\title{
Micropapillary Serous Borderline Ovarian Tumor in Early Pregnancy: Is Conservative Surgery Still a Viable Option?
}

\author{
Kazila Bhutiaa, d, Manisha Mathur $^{\mathrm{b}}$, Ieera Aggarwal ${ }^{\mathrm{c}}$
}

\begin{abstract}
With the advent of improved sonographic techniques, it is quite common to find ovarian masses during pregnancy. Most of these are benign and are usually managed conservatively. It is rare to find a malignant ovarian tumor in pregnancy with a reported incidence of 1 in 12,000 to 1 in 47,000 . Up to $8 \%$ of the adnexal masses could be borderline in nature. We present a case of a 29-year-old woman whose ultrasound in early first trimester ( 5 weeks) identified a solidcystic ovarian mass with a raised CA125, suspicious of ovarian malignancy. She did not have any symptoms arising from this mass. She underwent a laparotomy and unilateral salpingo-oophorectomy at 6 weeks gestation. Frozen section was reported as borderline papillary serous ovarian tumor and hence omentectomy was done to complete the staging. Histology confirmed micropapillary variant of serous borderline tumor with a few foci of stromal micro-invasion. She was staged as FIGO stage IC. She was managed conservatively with regular follow-up at the antenatal clinic. She delivered a healthy baby at term by cesarean section. She is currently free of disease and is on regular follow-up at the gynae-oncology clinic. We wish to highlight that although borderline ovarian tumors (BOTs) in general have a very favorable prognosis, those diagnosed during pregnancy can be associated with aggressive features. The micropapillary variant of the serous BOT has the potential of malignant transformation and may represent an early stage in the continuum of development of low-grade serous tumors of the ovary. These patients should be closely followed up to detect any recurrence or progressive disease early.
\end{abstract}

Keywords: Adnexal masses; Pregnancy; Borderline ovarian tumor; Micropapillary feature; Fertility sparing surgery

Manuscript accepted for publication August 16, 2016

aDivision of Obstetrics \& Gynecology, KK Women's and Children's Hospital, Singapore

bDepartment of Obstetrics \& Gynecology, KK Women's and Children's Hospital, Singapore

'Department of Gynae-oncology, KK Women's and Children's Hospital, Singapore

${ }^{\mathrm{d} C o r r e s p o n d i n g ~ A u t h o r: ~ K a z i l a ~ B h u t i a, ~ D i v i s i o n ~ o f ~ O b s t e t r i c s ~ \& ~ G y n e c o l-~}$ ogy, KK Women's and Children's Hospital, 100 Bukit Timah Road, Singapore 229899, Singapore. Email: Kazila.Bhutia@kkh.com.sg

doi: http://dx.doi.org/10.14740/jmc2617w

\section{Introduction}

It is not uncommon to find an ovarian mass in routine prenatal ultrasound scan but the likelihood of it being malignant is rare with a reported incidence of 1 in 12,000 to 1 in 47,000 [1]. The reported incidence of borderline ovarian tumor (BOT) in pregnant women is up to $8 \%$ [2-5] and $42 \%$ of these are serous subtype [6].

Various studies supported that serous BOT represents the precursor for low-grade serous carcinoma. This hypothesis is further substantiated by various studies demonstrating the presence of KRAS and BRAF gene mutation in both tumors [7-9]. Moreover, there is controversy about the nature of micropapillary subtype of serous BOT. The literature suggests that micropapillary subtype is more aggressive as compared to those without micropapillary pattern $[6,10,11]$. Burks et al reported similar clinicopathological features between micropapillary serous BOT and low-grade serous carcinoma [12]. They hypothesize that micropapillary serous BOT represents intermediate stage of tumor progression between serous BOT and low-grades serous carcinoma. Furthermore, May et al demonstrated that gene involved in mitogen-activated protein kinase (MAPK) signaling pathway showed higher expression in micropapillary serous BOT as compared to BOT without micropapillary, which further highlights the role of KRASBRAF-MEK-MAPK pathway in the progression to low-grade serous carcinoma [13].

Serous BOT with micropapillary projections may be considered as a potential precursor of low-grade serous carcinoma. It is therefore, this subset of patients who would require a more cautious approach towards management and follow-up.

\section{Case Report}

We report a case of a 29-year-old, gravida 3, para 2 woman with two previous lower segment cesarean sections who presented at 5 weeks pregnancy with nausea and vomiting. Her general physical, abdominal and gynecological examinations were unremarkable. A transvaginal ultrasound revealed a viable intrauterine pregnancy corresponding to 5 weeks gestation. In addition, a $9.4 \mathrm{~cm}$ left ovarian solid cystic mass with papillary projections was detected (Figs. 1,2). In view of high index of suspicion of malignancy, an MRI of pelvis was done. It confirmed a left ovarian mass measuring $10.5 \times 8.4 \times 7.7$ 


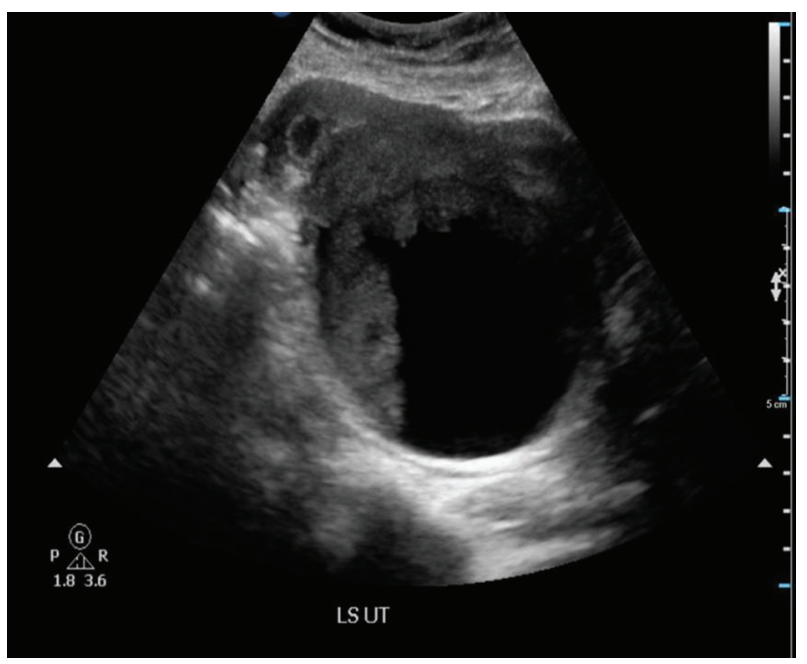

Figure 1. Ultrasound of pelvis showed a $9.4 \times 9.2 \times 7.3 \mathrm{~cm}$ complex solid cystic mass with solid component showing internal blood flow.

$\mathrm{cm}$, which was predominantly cystic with papillary projections and highly suggestive of malignancy (Fig. 3). There was no evidence of abdominal or pelvic lymphadenopathy, ascites or peritoneal disease. CA125 was raised at $104.8 \mathrm{U} / \mathrm{mL}$.

In view of ultrasound and MRI of pelvis findings, the working diagnosis was an ovarian malignancy or a BOT.

\section{Treatment}

In the preoperative period, the patient was reviewed by the gynae-oncology team and counseled about the advantages and disadvantages of proceeding with surgery in the first trimes-



Figure 2. Doppler ultrasound showing internal blood flow.

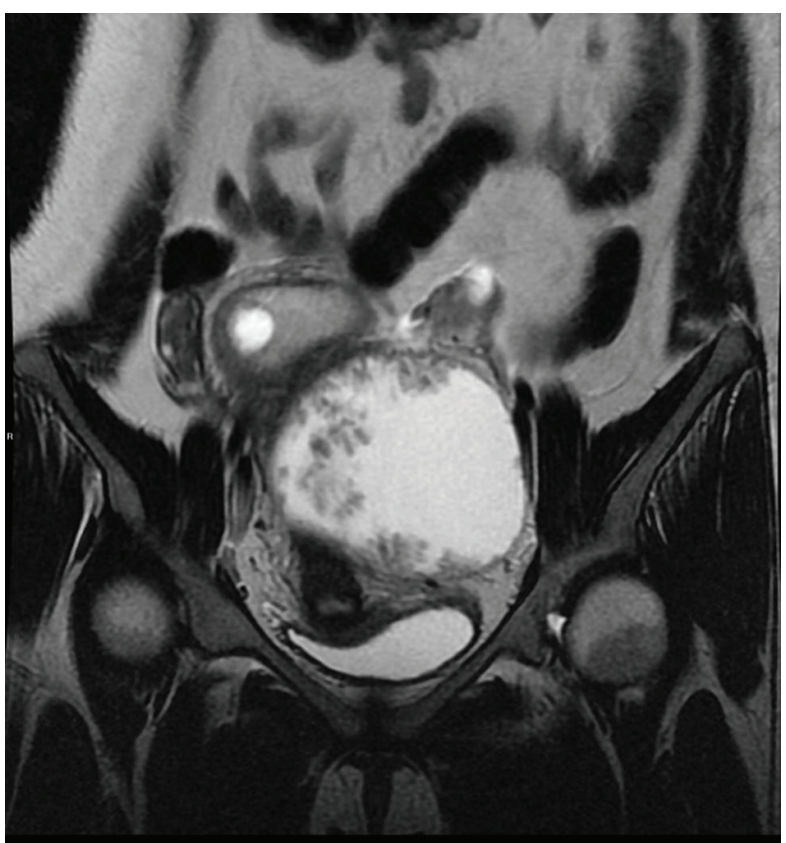

Figure 3. MRI of pelvis showed a large predominantly cystic lesion with papillary projection.

ter. Due to the high risk of malignancy and the risk of disease progression, the patient opted for early surgical intervention. However, due to religious beliefs, she wished to retain her fertility if the frozen section did not confirm a malignancy. She underwent a laparotomy at 6 weeks gestation. Intra-operatively, she was noted to have a $10 \mathrm{~cm}$ left ovarian mass adherent to the posterior ovarian fossa (Fig. 4). The uterus, right fallopian tube and the right ovary were normal in appearance. The rest of the intra-abdominal organs, including appendix and omentum were normal. During the adhesiolysis, there was rupture of the ovarian cyst with minimal spillage of cyst contents. Peritoneal washings were obtained and a left salpingo-oopherectomy was carried out. The frozen section of the left tube and ovary was reported as serous BOT with micropapillary features; hence in addition, an omentectomy was performed. As per the patient's wishes, the pregnancy was undisturbed and the contralateral tube and ovary were preserved. Subsequently, the patient re-

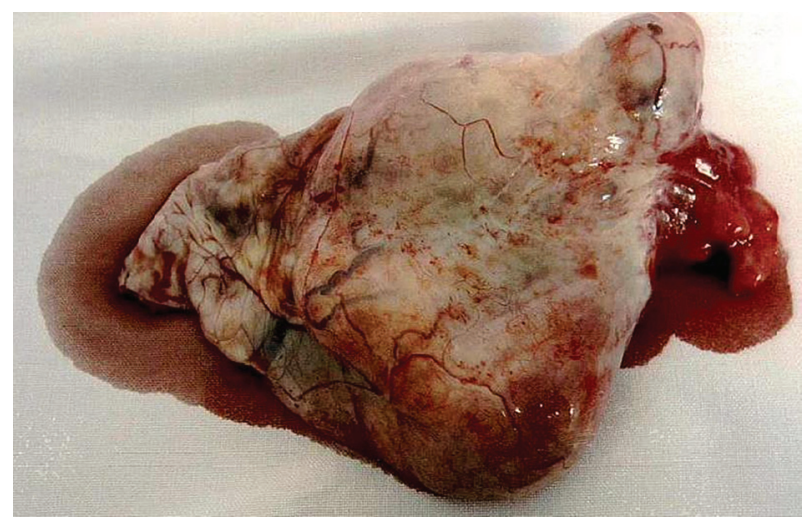

Figure 4. The $10 \mathrm{~cm}$ left ovarian mass. 
covered well and was discharged home on third postoperative day.

\section{Outcome and follow-up}

The final histology confirmed serous BOT FIGO stage IC, micropapillary variant with a few foci of stromal micro-invasion. There was no involvement of ovarian serosa and omentum and the peritoneal fluid was negative for malignant cells. The case was discussed at gynae-oncology multidisciplinary team meeting and conservative management with oncology followup was recommended. The rest of her antenatal period was uneventful. She underwent emergency cesarean section at 38 weeks 4 days period of gestation for two previous cesarean sections in labor. During cesarean section, intraperitoneal survey revealed normal right fallopian tube, ovary and appendix.

Although our patient had completed her family, she declined definitive surgery and opted for conservative management due to personal reasons. She is currently being followed up by the gynae-oncology team. At 18 months after primary surgery, she was noted to have a raised CA125 of $60.3 \mathrm{IU} / \mathrm{mL}$. She was asymptomatic with unremarkable physical examination. A CT scan of abdomen and pelvis showed no evidence of tumor recurrence. At her last follow-up with us at 24 months, her CA125 continued to be mildly elevated at $37.6 \mathrm{IU} / \mathrm{mL}$, but ultrasound of pelvis revealed normal contralateral tube and ovary and rest of the pelvis was normal.

\section{Discussion}

The incidence of adnexal mass in pregnancy is estimated to be between $0.19 \%$ and $2 \%$ [14-16]. However, the likelihood of this adnexal mass being malignant is rare, with reported incidence between 1 in 12,000 and 1 in 47,000 [14]. Epithelial ovarian cancer is the most common type of malignancy reported in pregnant women in contrast to non-pregnant younger population where germ cell tumor is more common $[1,14]$. Up to $8 \%$ of the adnexal masses could be borderline in nature [2-5] and $42 \%$ of these are serous in nature [6].

The majority of the patients with adnexal mass remain asymptomatic during pregnancy [1]. They are generally identified during first trimester routine ultrasonography [17]. Serum CA125 has a limited role to help to differentiate between benign and malignant tumors due to high false positive rate. Even in non-pregnant patient, the level of CA125 is elevated only in $50 \%$ of patients with stage I cases as compared to $80 \%$ of patients with advance stage [1, 18, 19]. Moreover, serum CA125 level may be elevated during the first trimester of pregnancy and starts falling by the end of the first trimester $[1,20]$. Transvaginal ultrasound is helpful in assessing the detailed characteristics of adnexal mass that may indicate the possibility of mass being malignant. The complex ovarian mass, papillary projections, septation, and increased vascularity are some features associated with an increased risk of malignancy $[1,21]$. In our patient, a suspicious complex ovarian cyst with papillary projections and internal vascularity was seen at a transvaginal scan. Due to high index of suspicion of ovarian malignancy, MRI of abdomen and pelvis was performed. MRI of pelvis is preferred in pregnant patients due to lack of radiation exposure.

The standard treatment for BOT is hysterectomy with bilateral salphingoophorectomy, peritoneal washing \pm appendicectomy/omentectomy. However, median age of diagnosis of BOT is about 40 years with more than one-third being in the reproductive age group $[17,22]$. Hence, the role of conservative surgery preserving childbearing potential is an important issue. The tumors have an indolent natural history and show excellent survival rate. Survival for women with early stage (FIGO I-II) disease is virtually $100 \%$ and between $86 \%$ and $92 \%$ in more advanced stage (FIGO III-IV) [23].

Kashima et al have shown promising outcomes for the fertility sparing conservative surgery if patients wish to retain their fertility [24]. Similarly, Battaglia et al presented a rare case of a 30-year-old patient with stage IC ovarian cancer with borderline tumor on contralateral ovary, who underwent conservative debulking surgery followed by adjuvant chemotherapy. The patient conceived successfully 5 years after surgery and had no evidence of disease recurrence after 5 years of follow-up [22].

Available literature is divided on laparotomy or laparoscopy as the modality of surgical intervention. Some suggest that laparoscopy stands out on account of less blood loss, less postoperative pain and less postoperative hospital stay, a faster recovery and less pelvic adhesion [25]. On the contrary, Ilancheran et al believed that midline laparotomy is more appropriate even in pregnant women as it gives better visualization of the peritoneal cavity for cancer implants with less manipulation of the gravid uterus [26]. In this patient, we proceeded with midline laparotomy as radiological findings suggested high possibility of malignancy.

Similarly, the actual timing of surgery has also been a matter of debate. Some researchers believe that a strong suspicion of malignancy on imaging and abnormal tumor markers should merit early surgical intervention [27, 28]. However, Marret et al suggested that the surgical intervention, which might include conservative surgery or debulking surgery along with chemotherapy, could be deferred until after 15 weeks of gestation [29]. By second trimester, pregnancy reaches a stable state and surgery can be carried out without affecting disease progression [29, 30]. Given a strong suspicion of an ovarian cancer, following discussion with patient, we proceeded with surgery in the first trimester with luteal phase support.

The intra-operative frozen section in the present case was reported as borderline serous tumor with papillary projections. The final histopathology was serous borderline tumor and micropapillary variant with few foci of stromal micro-invasion. Even though the right ovary appeared grossly normal in the present case, the serous BOTs are bilateral in about one-third of cases and one-third of cases are associated with peritoneal implants $[23,27,31,32]$. According to Fauvet et al, BOTs diagnosed during pregnancy exhibit a high incidence of aggressive features and are rarely completely staged initially [6]. Women with serous BOT without invasive implants have 10year survival of $95 \%$ compared with $60-70 \%$ for women with 
invasive implants [32]. In our case, having BOT confined to one ovary with no invasive implants and thus we anticipate an excellent survival.

Various studies supported that serous BOT represents the precursor of low-grade serous carcinoma. This hypothesis is further substantiated by various literatures demonstrating the presence of KRAS and BRAF gene mutation in both tumors [7-9]. Moreover, the micropapillary pattern, which was detected in this patient on final histopathological report, has been matter of concern as it could indicate poorer prognosis for recurrence. The literature suggests that micropapillary subtype is more aggressive as compared to those without micropapillary pattern $[6,10,11]$. Longacre et al have also reported that in addition to high-risk FIGO stage (III, IV) and invasive implants, micropapillary architecture is associated with high recurrence risk and/or progressive nature of serous BOTs [33].

Kurman et al reported similar clinicopathological features between micropapillary serous BOT and low-grade serous carcinoma [12]. They hypothesize that micropapillary serous BOT may represent intermediate stage of tumor progression between serous BOT and low-grade serous carcinoma. Furthermore, May et al demonstrated that gene involved in MAPK signaling pathway showed higher expression in micropapillary serous BOT as compared to BOT without micropapillarity, which further highlights the role of KRAS-BRAFMEK-MAPK pathway in the progression to low-grade serous carcinoma [13].

\section{Conclusions}

Due to aggressive course of micropapillary serous BOT and increased incidence of malignant transformation to low-grade serous tumor as compared to other borderline tumors, it is therefore important to have long-term follow-up at gynae-oncology center for early diagnosis of recurrence. Considering radical surgery once the family is completed is worth a consideration in all patients with micropapillary serous BOT.

\section{References}

1. Aggarwal P, Kehoe S. Ovarian tumours in pregnancy: a literature review. Eur J Obstet Gynecol Reprod Biol. 2011;155(2):119-124.

2. Bromley B, Benacerraf B. Adnexal masses during pregnancy: accuracy of sonographic diagnosis and outcome. J Ultrasound Med. 1997;16(7):447-452; quiz 453-444.

3. Whitecar MP, Turner S, Higby MK. Adnexal masses in pregnancy: a review of 130 cases undergoing surgical management. Am J Obstet Gynecol. 1999;181(1):19-24.

4. Ueda M, Ueki M. Ovarian tumors associated with pregnancy. Int J Gynaecol Obstet. 1996;55(1):59-65.

5. Sherard GB, 3rd, Hodson CA, Williams HJ, Semer DA, Hadi HA, Tait DL. Adnexal masses and pregnancy: a 12year experience. Am J Obstet Gynecol. 2003;189(2):358362; discussion 362-353.

6. Fauvet R, Brzakowski M, Morice P, Resch B, Marret H, Graesslin O, Darai E. Borderline ovarian tumors diag- nosed during pregnancy exhibit a high incidence of aggressive features: results of a French multicenter study. Ann Oncol. 2012;23(6):1481-1487.

7. Singer G, Oldt R, 3rd, Cohen Y, Wang BG, Sidransky D, Kurman RJ, Shih Ie M. Mutations in BRAF and KRAS characterize the development of low-grade ovarian serous carcinoma. J Natl Cancer Inst. 2003;95(6):484-486.

8. Bonome T, Lee JY, Park DC, Radonovich M, Pise-Masison C, Brady J, Gardner GJ, et al. Expression profiling of serous low malignant potential, low-grade, and high-grade tumors of the ovary. Cancer Res. 2005;65(22):1060210612.

9. Malpica A, Deavers MT, Lu K, Bodurka DC, Atkinson EN, Gershenson DM, Silva EG. Grading ovarian serous carcinoma using a two-tier system. Am J Surg Pathol. 2004;28(4):496-504.

10. Shih Ie M. Ovarian serous low malignant potential (borderline) tumor - does "micropapillary" matter? Gynecol Oncol. 2010;117(1):1-3.

11. Cho KR, Shih Ie M. Ovarian cancer. Annu Rev Pathol. 2009;4:287-313.

12. Burks RT, Sherman ME, Kurman RJ. Micropapillary serous carcinoma of the ovary. A distinctive low-grade carcinoma related to serous borderline tumors. Am J Surg Pathol. 1996;20(11):1319-1330.

13. May T, Virtanen C, Sharma M, Milea A, Begley H, Rosen $\mathrm{B}$, Murphy KJ, et al. Low malignant potential tumors with micropapillary features are molecularly similar to low-grade serous carcinoma of the ovary. Gynecol Oncol. 2010;117(1):9-17.

14. Leiserowitz GS, Xing G, Cress R, Brahmbhatt B, Dalrymple JL, Smith LH. Adnexal masses in pregnancy: how often are they malignant? Gynecol Oncol. 2006;101(2):315321.

15. Bernhard LM, Klebba PK, Gray DL, Mutch DG. Predictors of persistence of adnexal masses in pregnancy. Obstet Gynecol. 1999;93(4):585-589.

16. Marino T, Craigo SD. Managing adnexal masses in pregnancy. Contemp Ob/Gyn. 2000;45(5):130-143.

17. Park JY, Kim DY, Kim JH, Kim YM, Kim YT, Nam JH. Surgical management of borderline ovarian tumors: The role of fertility-sparing surgery. Gynecol Oncol. 2009;113(1):75-82.

18. Vuento MH, Stenman UH, Pirhonen JP, Makinen JI, Laippala PJ, Salmi TA. Significance of a single CA 125 assay combined with ultrasound in the early detection of ovarian and endometrial cancer. Gynecol Oncol. 1997;64(1):141-146.

19. Einhorn N, Sjovall K, Knapp RC, Hall P, Scully RE, Bast RC, Jr., Zurawski VR, Jr. Prospective evaluation of serum CA 125 levels for early detection of ovarian cancer. Obstet Gynecol. 1992;80(1):14-18.

20. CA-125. Test interpretation. Available from: URL: http:// www.clinlabnavigator.com/Tests/CA125.html.

21. Nossov V, Amneus M, Su F, Lang J, Janco JM, Reddy ST, Farias-Eisner R. The early detection of ovarian cancer: from traditional methods to proteomics. Can we really do better than serum CA-125? Am J Obstet Gynecol. 2008;199(3):215-223. 
22. Battaglia F, Plotti F, Zullo MA, Panici PB, Plotti G. Successful pregnancy after conservative surgery for stage IC ovarian cancer with serous borderline tumor on controlateral ovary: a case report. Gynecol Oncol. 2006;100(3):612-614.

23. Trope CG, Kaern J, Davidson B. Borderline ovarian tumours. Best Pract Res Clin Obstet Gynaecol. 2012;26(3):325-336.

24. Kashima K, Yahata T, Fujita K, Tanaka K. Outcomes of fertility-sparing surgery for women of reproductive age with FIGO stage IC epithelial ovarian cancer. Int J Gynaecol Obstet. 2013;121(1):53-55.

25. Chen L, Ding J, Hua K. Comparative analysis of laparoscopy versus laparotomy in the management of ovarian cyst during pregnancy. J Obstet Gynaecol Res. 2014;40(3):763-769.

26. Ilancheran A, Low J, Ng JS. Gynaecological cancer in pregnancy. Best Pract Res Clin Obstet Gynaecol. 2012;26(3):371-377.

27. Bignardi T, Condous G. The management of ovarian pathology in pregnancy. Best Pract Res Clin Obstet Gynaecol. 2009;23(4):539-548.

28. Yen CF, Lin SL, Murk W, Wang CJ, Lee CL, Soong
YK, Arici A. Risk analysis of torsion and malignancy for adnexal masses during pregnancy. Fertil Steril. 2009;91(5):1895-1902.

29. Marret H, Lhomme C, Lecuru F, Canis M, Leveque J, Golfier F, Morice P. Guidelines for the management of ovarian cancer during pregnancy. Eur J Obstet Gynecol Reprod Biol. 2010;149(1):18-21.

30. Grimm D, Woelber L, Trillsch F, Keller-v Amsberg G, Mahner S. Clinical management of epithelial ovarian cancer during pregnancy. Eur J Cancer. 2014;50(5):963971.

31. Morice P, Uzan C, Gouy S, Verschraegen C, Haie-Meder C. Gynaecological cancers in pregnancy. Lancet. 2012;379(9815):558-569.

32. Seidman JD, Horkayne-Szakaly I, Haiba M, Boice CR, Kurman RJ, Ronnett BM. The histologic type and stage distribution of ovarian carcinomas of surface epithelial origin. Int J Gynecol Pathol. 2004;23(1):41-44.

33. Longacre TA, McKenney JK, Tazelaar HD, Kempson RL, Hendrickson MR. Ovarian serous tumors of low malignant potential (borderline tumors): outcome-based study of 276 patients with long-term ( $>$ or $=5$-year) follow-up. Am J Surg Pathol. 2005;29(6):707-723. 